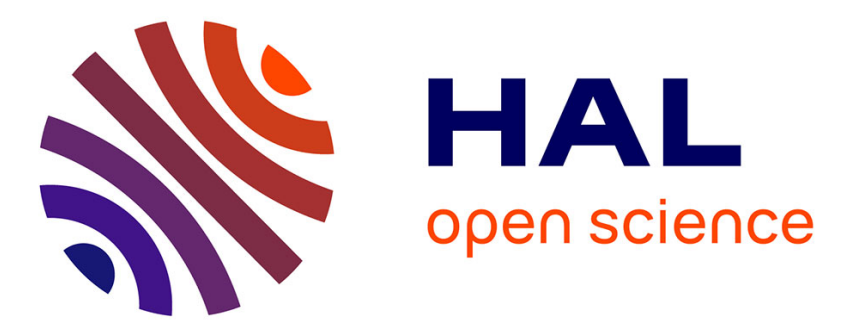

\title{
Mesure directe par élargissement stark des raies de l'hydrogène de la densité de courant dans un gaz ionisé ou règne un champ macroscopique quasi uniforme
} F. Bastien, E. Marode

\section{- To cite this version:}

F. Bastien, E. Marode. Mesure directe par élargissement stark des raies de l'hydrogène de la densité de courant dans un gaz ionisé ou règne un champ macroscopique quasi uniforme. Revue de Physique Appliquée, 1977, 12 (8), pp.1121-1125. 10.1051/rphysap:019770012080112100 jpa-00244288

\section{HAL Id: jpa-00244288 https://hal.science/jpa-00244288}

Submitted on 1 Jan 1977

HAL is a multi-disciplinary open access archive for the deposit and dissemination of scientific research documents, whether they are published or not. The documents may come from teaching and research institutions in France or abroad, or from public or private research centers.
L'archive ouverte pluridisciplinaire HAL, est destinée au dépôt et à la diffusion de documents scientifiques de niveau recherche, publiés ou non, émanant des établissements d'enseignement et de recherche français ou étrangers, des laboratoires publics ou privés. 


\title{
MESURE DIRECTE PAR ÉLARGISSEMENT STARK DES RAIES DE L'HYDROGËNE DE LA DENSITÉ DE COURANT DANS UN GAZ IONISÉ OU RẼGNE UN CHAMP MACROSCOPIQUE QUASI UNIFORME (*)
}

\author{
F. BASTIEN et E. MARODE
}

\author{
Laboratoire de Physique des décharges, C. N. R. S., Ecole Supérieure d'Electricité, \\ Plateau du Moulon, 91190 Gif-sur-Yvette, France
}

(Reçu le 30 décembre 1976, accepté le 20 avril 1977)

\begin{abstract}
Résumé. - L'analyse théorique de l'élargissement de raies de l'hydrogène pour un gaz ionisé où règne un champ macroscopique $F_{\mathrm{c}}$ (champ de charge d'espace dans les décharges prédisruptives par exemple) montre que l'élargissement ne dépend plus seulement de la densité électronique $N_{\text {e }}$ mais également de la valeur du champ macroscopique $F_{\mathrm{c}}$. Une conséquence est développée ici : dans un certain domaine, l'élargissement est pratiquement fonction du produit $N_{\mathrm{e}} F_{\mathrm{c}}$. Il devient alors possible d'évaluer directement la densité de courant en introduisant une petite quantité d'hydrogène dans un gaz où la mobilité des particules est connue. Un exemple d'application est donné (décharge couronne).
\end{abstract}

\begin{abstract}
A theorical analysis of the hydrogen line broadening for an ionised gas in the presence of a macroscopic field $F_{\mathrm{c}}$ (for example, a space charge field within a pre-breakdown discharge) shows that the broadening depends not only on the charged particle density $N_{\mathrm{e}}$ but also on the macroscopic field $F_{\mathrm{c}}$. A consequence developed here is that in a certain range the broadening is pratically dependant only on the product $N_{\mathrm{e}} F_{\mathrm{c}}$. It is thus possible to evaluate directly the current density by measuring the line broadening induced in hydrogen introduced into a gas in minute concentration when the mobilities of particles in that gas are known. An example of an application is given (corona discharge).
\end{abstract}

1. Introduction. - Dans les décharges électriques les paramètres faciles à mesurer sont le courant et la tension appliquée. Malheureusement ces paramètres ne permettent pas de déterminer les mécanismes importants du phénomène. Par contre les paramètres plus fondamentaux, comme la densité électronique, la densité de courant et le champ électrique sont d'un accès difficile.

Une méthode pour atteindre la densité électronique est de mesurer l'élargissement Stark des raies émises par l'hydrogène atomique. Cependant, pour les milieux ionisés créés par les décharges électriques une difficulté importante apparaît.

En effet, dans les décharges prédisruptives, si le transfert d'énergie électrons-particules lourdes reste faible, il n'y a pas d'ionisation thermique, la conductivité reste assez basse pour permettre la présence d'un champ macroscopique.

(*) Communication présentée au Congrès National de Physique des Plasmas, Paris, 6-10 décembre 1976.
L'atome émetteur se trouve alors soumis à un champ statique qui se superpose au micro-champ aléatoire généralement considéré seul.

L'élargissement n'est plus alors uniquement fonction de la densité $N_{\mathrm{e}}$ des particules chargées mais aussi du champ de charge d'espace $F_{\text {c. }}$.

Nous avons élaboré la théorie de l'élargissement Stark correspondant à ces conditions (voir réf. [1]).

Nous allons discuter ici des conséquences de cette théorie en montrant notamment que l'élargissement Stark en présence d'un champ macroscopique est lié à la densité de courant plutôt qu'à la densité des particules chargées.

2. Elargissement des raies de l'hydrogène dans un milieu ionisé en présence d'un champ macroscopique. Considérons une transition entre les niveaux $\mid \mathrm{a}>$ et $\mid \mathrm{b}>$ séparée dans l'action d'un champ électrique en sous-niveau $|\alpha\rangle$ et $\mid \beta>$ respectivement.

En raison des collisions électroniques de chaque raie $\omega_{\alpha \beta}$ présente un profil donné dans le formalisme 
de Griem, Kolb et Shen [2] (approximation d'impact) et pour une radiation polarisée dans une direction $\mathbf{e}$ perpendiculaire à une direction d'observation $\mathbf{k}$ par

$$
\begin{aligned}
\mathcal{F}_{\mathrm{e}}(\omega, F)= & \frac{\mathrm{d} J_{\mathrm{c}}(\omega, F)}{\mathrm{d} \Omega_{\text {obs. }}} \\
= & \pi^{-1} \sum_{\alpha^{\prime} \alpha^{\prime \prime} \beta} \operatorname{Re}<\beta \mid \text { e.r }\left|\alpha^{\prime} \quad \alpha^{\prime \prime}\right| \mathbf{e} . \mathbf{r} \mid \beta>\times \\
& \times<\alpha^{\prime \prime}\left|\left[\mathrm{i} \Delta \omega_{\alpha^{\prime \prime} \beta}(F)-\varphi_{0}\right]^{-1}\right| \alpha^{\prime}>\quad(1)
\end{aligned}
$$

dans cette expression nous avons :

r: position vectorielle de l'électron de l'atome $H$ $\mathrm{d} \Omega_{\text {obs. }}$ : angle solide d'observation dans la direction $\mathbf{k}$ $\varphi_{\mathrm{a}}=$ opérateur collisions voir (2) et (3).

$\Delta \omega_{\alpha^{\prime \prime} \beta}(F)=\omega-\omega_{\alpha^{\prime \prime} \beta}=\omega-\omega_{\mathrm{ab}}-\left(\omega_{\mathrm{a}^{\prime \prime} \beta}-\omega_{\mathrm{ab}}\right)$ avec $\hbar \omega_{\mathrm{ab}}=E_{0}^{\mathrm{a}}-E_{0}^{\mathrm{b}}$ où $E_{0}^{\mathrm{a}}$ et $E_{0}^{\mathrm{b}}$ sont les énergies des niveaux non perturbés $|\mathrm{a}\rangle$ et $|\mathrm{b}\rangle$.

Cette expression suppose l'équipartition de la population des sous-niveaux de l'état initial quasi réalisée en cas d'équilibre thermodynamique local (E. T. L.). En fait l'existence d'un champ macroscopique est généralement associée à une situation hors équilibre. Cependant les fréquences de collisions électron-atome d'hydrogène assurant le passage de l'atome excité d'un sous-niveau à un autre, estimé à partir de $\varphi_{\mathrm{a}}\left(\simeq 2,5 \times 10^{10} \mathrm{~s}^{-1}\right.$ pour $\mathrm{H}_{\alpha}, 8 \times 10^{10} \mathrm{~s}^{-1}$ pour $\mathrm{H}_{\beta}$ avec $T_{\mathrm{e}}=10^{4} \mathrm{~K}$ et $N_{\mathrm{e}}=10^{15} \mathrm{~cm}^{-3}$ ) sont très supérieures aux autres fréquences de collisions avec l'atome d'hydrogène et correspondent à un temps entre deux collisions inférieur aux durées de vie radiatives.

La répaitition des populations dans les sous-niveaux est donc essentiellement gouvernée par les chocs électroniques; il s'en suit que même si la population globale du niveau considéré est différente de celle qu'elle acquiert à l'E. T. L., la quasi-équipartition doit être réalisée car la proximité des énergies entre sousniveaux entraîne la quasi-égalité des sections efficaces directes et inverses.

On peut sinterroger sur la forme de la distribution électronique supposée maxwellienne dans l'évaluation de $\varphi_{\mathrm{a}}$. Cette approximation semble raisonnable. En effet, d'une part la valeur de $\varphi_{\mathrm{a}}$ dépend peu de la forme de la queue de distribution et d'autre part si l'hydrogène est introduit dans un gaz moléculaire les nombreux chocs inélastiques tendent à dépeupler la queue de distribution au bénéfice de la partie centrale si bien qu'une distribution maxwellienne est mieux adaptée à ce cas qu'une distribution de Druveystein qui ne rend compte que du recul du aux chocs élastiques (cas des gaz rares).

En l'absence de champ macroscopique la distribution des champs s'appliquant sur l'atome d'hydrogène est isotrope si bien que l'émission est isotrope et s'obtient en intégrant la relation (1) sur l'espace et les champs.

Dans le cas que nous considérons la situation est différente. En effet, la figure 1 donne un exemple de

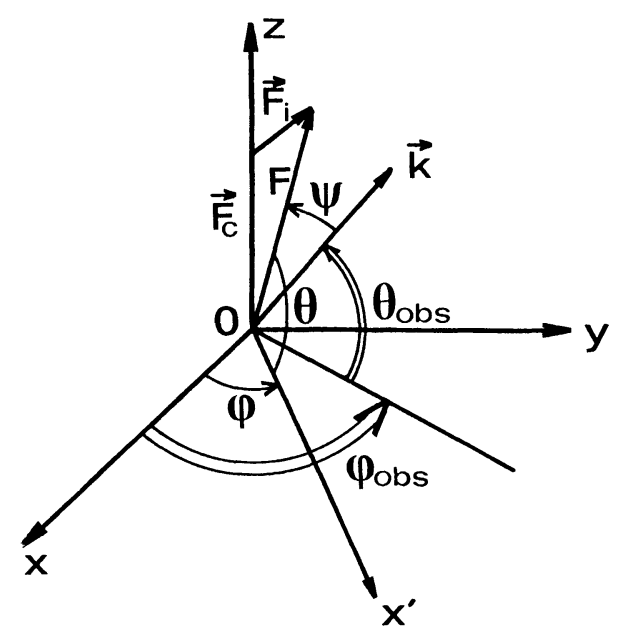

Fig. 1. - Système de coordonnées lié au champ statique $\mathbf{F}_{\mathbf{c}}$. [Coordinate system bound to the resulting field $\mathbf{F}_{\mathrm{c}}$.]

composition du champ macroscopique $F_{\mathrm{c}}$ avec le champ microscopique $F_{\mathrm{i}}$. Chaque atome émetteur est soumis à un même champ $F_{\mathrm{c}}$ mais à un champ $F_{\mathrm{i}}$ différent dont la distribution est isotrope. Les champs résultants $F$ obéissent donc à une distribution anisotrope. Une analyse angulaire (rôle différent des composants $\pi$ et $\sigma$ ) ainsi que la détermination des champs résultants conduit finalement à l'expression :

$$
\begin{aligned}
& L(\omega)=\int_{0}^{\infty} \mathrm{d} F\left\{\mathscr{F}_{Z}(\omega, F) \int_{-\pi / 2}^{+\pi / 2} \mathrm{~d} \theta \frac{W_{\mathrm{i}}\left[\left(F^{2}+F_{\mathrm{c}}^{2}-2 F F_{\mathrm{c}} \sin \theta\right)^{1 / 2}\right]}{2} \frac{F^{2}}{F^{2}+F_{\mathrm{c}}^{2}-2 F F_{\mathrm{c}} \sin \theta} \times\right. \\
& \times \cos \theta\left[1-\frac{\cos ^{2} \theta \cos ^{2} \theta_{\text {obs. }}}{2}-\sin ^{2} \theta \sin ^{2} \theta_{\text {obs. }}\right] \\
& +\mathscr{F}_{X}(\omega, F) \int_{-\pi / 2}^{+\pi / 2} \frac{W_{\mathrm{i}}\left[\left(F^{2}+F_{\mathrm{c}}^{2}-2 F_{\mathrm{c}} F \sin \theta\right)^{1 / 2}\right]}{2} \frac{F^{2}}{F^{2}+F_{\mathrm{c}}^{2}-2 F F_{\mathrm{c}} \sin \theta} \cos \theta \\
& \left.\times\left[1+\frac{\cos ^{2} \theta \cos ^{2} \theta_{\text {obs. }}}{2}+\sin ^{2} \theta \sin ^{2} \theta_{\text {obs. }}\right] \mathrm{d} \theta\right\} \text {. }
\end{aligned}
$$


Dans cette formule $\theta$ repère $F$ par rapport à $F_{\mathrm{c}}$, $\theta_{\text {obs. }}$ repère $k$ par rapport à $F_{\mathrm{c}}$ (voir Fig. 1),

$\mathscr{F}_{X}$ et $\mathscr{F}_{Z}$ s'obtiennent à partir de $\mathfrak{F}_{e}$ en remplaçant e.r respectivement par $X$ et $Z$; ces quantités apparaissent lors de l'analyse angulaire.

$X, Y, Z$ composantes du vecteur $\mathbf{r}$;

$W_{\mathbf{i}}$ est une fonction de distribution du microchamp ionique (nous avons utilisé celle donnée par Hooper [4]).

La symétrie cylindrique du problème fait disparaître

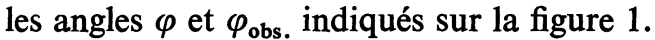

L'expression (2) montre que le profil dépend de la direction d'observation et que de plus le profil peut se mettre sous la forme

$$
L(\omega)=\cos ^{2} \theta_{\text {obs. }} L_{\perp}(\omega)+\sin ^{2} \theta_{\text {obs. }} L_{\|}(\omega)
$$

où $L_{\perp}$ profil pour une direction d'observation perpendiculaire champ $F_{\mathrm{c}}, L_{\|}$profil pour une direction d'observation parallèle au champ $F_{\mathrm{e}}$.

La figure 2 donne un exemple de profils $L_{\perp}$ et $L_{\|}$.

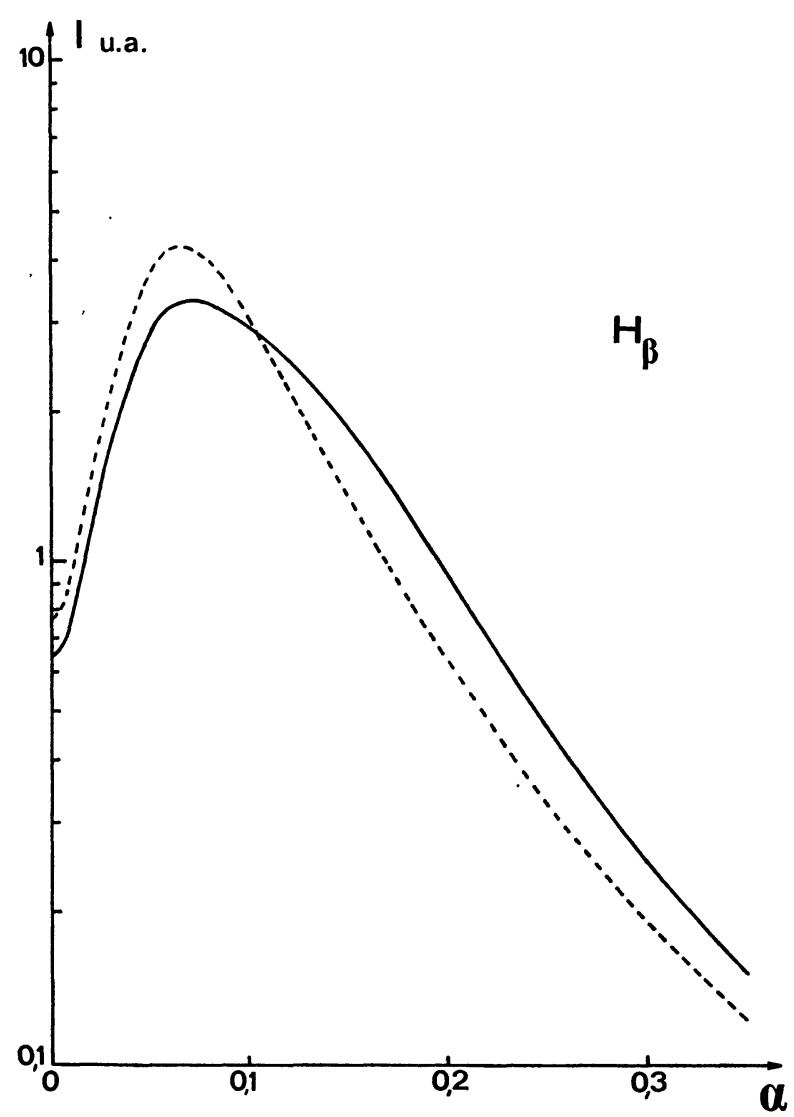

Fig. 2. - Comparaison des profils $L_{\perp}$ et $L_{\|}$pour $\mathrm{H}_{\beta}$ avec $N_{\mathrm{e}}=1016 \mathrm{~cm}^{-3}, T_{\mathrm{e}}=20000 \mathrm{~K}, p_{\mathrm{c}}=3\left(L_{\perp} \perp ; L_{\|^{----}}\right)$. [Comparison of $L_{\perp}$ and $L_{\|}$profiles for $\mathrm{H}_{\alpha}$ and $\mathrm{H}_{\beta}$ with $N_{\mathrm{e}}=1016 \mathrm{~cm}^{-3}, T_{\mathrm{e}}=20000 \mathrm{~K}, p_{\mathrm{c}}=3\left(L_{\perp} \longrightarrow, L_{\|^{---}}\right)$.]

L'étude de l'action d'une distribution du microchamp non isotrope a déjà été abordée par V. O. Jensen [5]. Cet auteur cependant développe son analyse dans le cadre non quantique de la théorie ancienne de
Holtzmark et dans un cas d'anisotropie très différent. Notons par ailleurs que indépendamment de ce travail un formalisme voisin a été utilisé par C. Deutsch et G. Gekefi [6] pour étudier l'élargissement de Lyman $\alpha$ dans un plasma turbulent.

3. Détermination de la densité de courant. - Nous voyons que le profil obtenu pour une direction d'observation donnée (par exemple perpendiculaire au champ statique) dépend de façon complexe de $N_{\mathrm{e}}$ et $F_{\mathrm{c}}$, ou ce qui revient au même de $N_{\mathrm{e}}$ et $p_{\mathrm{c}}=F_{\mathrm{c}} / F_{0}$ avec $F_{\mathrm{c}}=2,6 e N_{\mathrm{e}}^{2 / 3}$ (CGSES) champ de Holtzmark caractéristique de la distribution du microchamp. Notons de plus que le profil est très faiblement dépendant de la température électronique et que $N_{\mathrm{e}}=N_{\mathrm{i}}$ (égalité des densités électroniques et ioniques).

Sur les exemples d'élargissement de $\mathrm{H}_{\alpha}$ et $\mathbf{H}_{\beta}$ donnés par les figures 3 et 4 , on voit que pour $0 \leqslant p_{c}<1$ la raie reste à peu près identique à elle-même ; par contre, pour $p_{\mathrm{c}}>1$ l'élargissement augmente rapidement.

Dans ce dernier cas, on voit que l'élargissement est dû à la fois à l'action des particules chargées et du

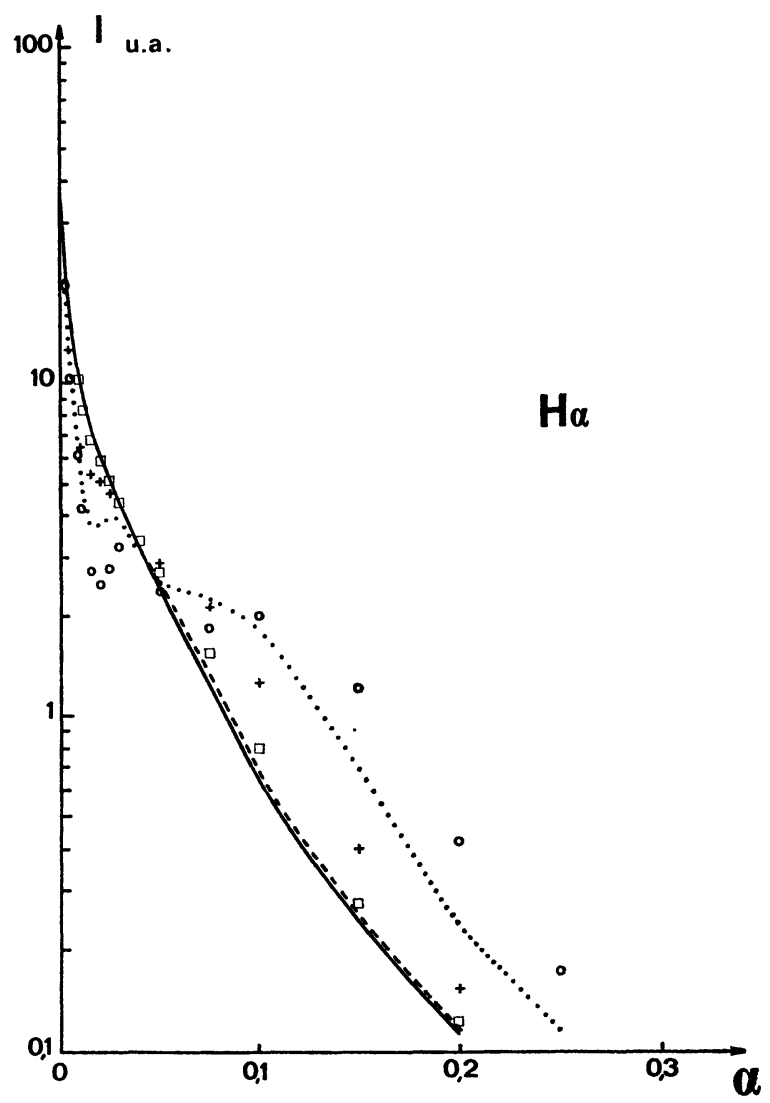

Fig. 3. - Elargissement de $H_{\alpha}$ en fonction du paramètre $\alpha=\Delta \lambda(\AA) / F_{0}$ (C. G. S.) pour $N_{\mathrm{e}}=10^{15} \mathrm{~cm}^{3} \cdot T_{\mathrm{e}}=20000 \mathrm{~K}$ et diverses valeurs du paramètre $p_{\mathrm{c}}=F_{\mathrm{c}} / F_{0}\left(p_{\mathrm{c}}=0\right.$ $p_{\mathrm{c}}=0,5 \ldots ; p_{\mathrm{c}}=1 * * * *, p_{\mathrm{c}}=2+++; p_{\mathrm{c}}=3 \ldots$; $p_{\mathrm{c}}=4$ ०००).

[ $\mathrm{H}_{\alpha}$ broadening as a function of $\alpha=\Delta \lambda(\AA) / F_{0}$ (C. G. S.) for $N_{\mathrm{e}}=10^{15} \mathrm{~cm}^{-3}, T_{\mathrm{e}}=20000 \mathrm{~K}$ and various values of the anisotropy factor $p_{\mathrm{c}}=F_{\mathrm{c}} / \boldsymbol{F}_{0}$.] 


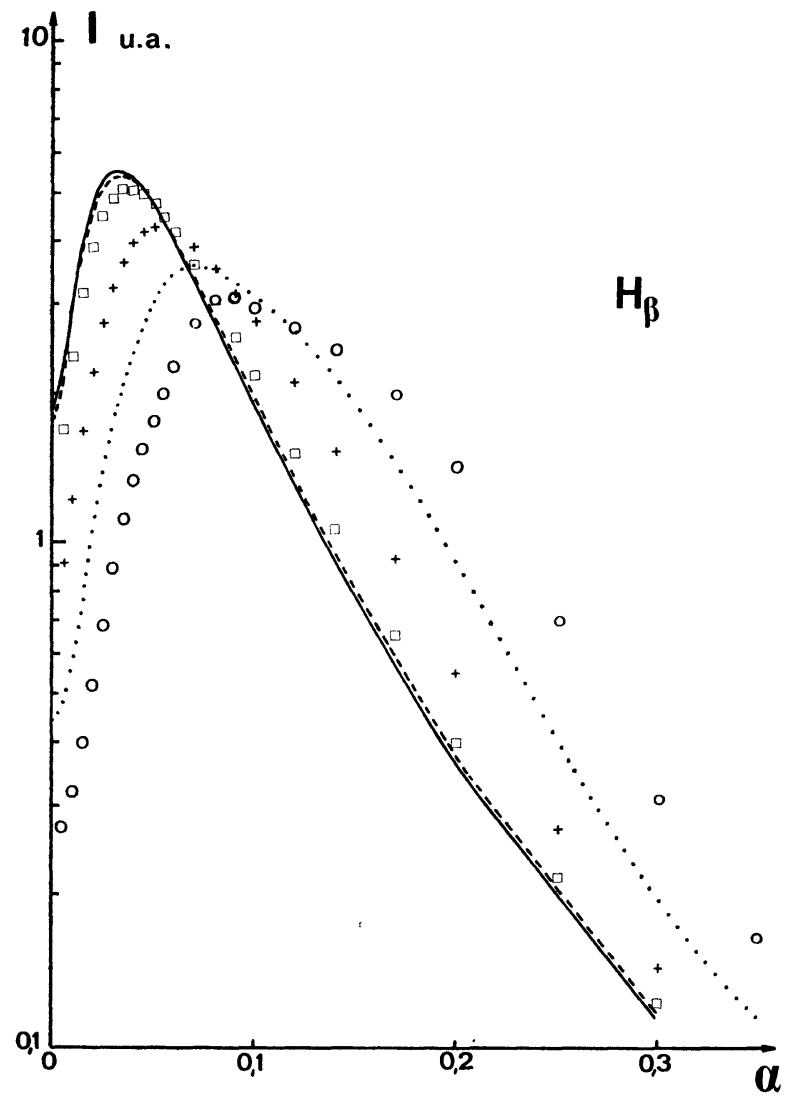

Fig. 4. - Elargissement de $\mathbf{H}_{\beta}$ (même légende que la figure 3). $\left[\mathrm{H}_{\beta}\right.$ broadening (caption as figure. 3).]

champ macroscopique. Afin de connaître l'action respective des paramètres $N_{\mathrm{e}}$ et $F_{\mathrm{c}}$ sur l'élargissement, nous avons comparé les profils obtenus pour divers couples $N_{\mathrm{e}} F_{\mathrm{c}}$. Dans le domaine $1 \leqslant p_{\mathrm{c}} \leqslant 4$ le résultat marquant est de constater que les profils sont sensiblement les mêmes quand la valeur du produit $N_{\mathrm{e}} F_{\mathrm{c}}$ reste constante. Des exemples de comparaisons sont donnés sur la figure 5 .

De la comparaison des profils théoriques et expérimentaux on peut donc tirer une valeur de $N_{\mathrm{e}} F_{\mathrm{c}}$. Dans le cas de $\mathbf{H}_{\beta}$ plus utilisable, car la raie est beaucoup plus élargie que $\mathbf{H}_{\alpha}$ dans les mêmes conditions, une méthode de comparaison plus rapide est possible. Nous avons tracé $\Delta \lambda$ (demi-élargissement à la moitié du maximum) en fonction de $N_{\mathrm{e}} F_{\mathrm{c}}$ pour $1<p_{\mathrm{c}}<4$. Sur la figure 6 , nous constatons qu'il existe une bonne corrélation entre l'élargissement et le produit $N_{\mathrm{e}} F_{\mathrm{c}}$.

L'intérêt de la détermination du produit $N_{\mathrm{e}} F_{\mathrm{c}}$ est qu'elle conduit à la valeur de la densité de courant $j$; en effet, si nous considérons la mobilité des particules chargées $\mu_{\mathrm{i}}$ et $\mu_{\mathrm{e}}$, nous avons $j=e\left(\mu_{\mathrm{e}}+\mu_{\mathrm{i}}\right) N_{\mathrm{e}} F_{\mathrm{c}}$ avec l'hypothèse de neutralité électrique, en fait $j \simeq j_{\mathrm{e}}=e \mu_{\mathrm{e}} N_{\mathrm{e}} F_{\mathrm{c}}$ car $\mu_{\mathrm{e}} \gg \mu_{\mathrm{i}}$.

Pratiquement, l'élargissement Stark est obtenu à partir du profil expérimental après déconvolution des élargissements instrumental et Doppler.

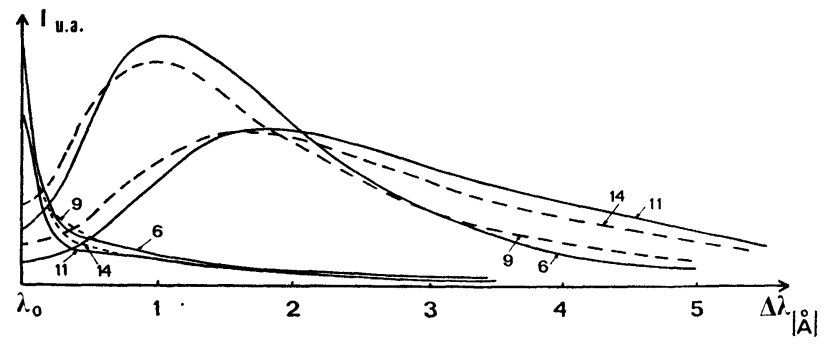

Fig. 5. - Comparaison de profils $H_{\alpha}$ et $H_{\beta}$ pour diverses valeurs du produit $N_{\mathrm{e}} F_{\mathrm{c}}$.

$$
\begin{aligned}
& \text { Courbes } 6-N_{\mathrm{e}} F_{\mathrm{c}}=0,79 \times 10^{17} \text { C. G. S. E. S } \\
& N_{\mathrm{e}}=2 \times 10^{15} \mathrm{~cm}^{-3} \quad p_{\mathrm{c}}=2 \\
& 9-N_{\mathrm{e}} F_{\mathrm{c}}=0,79 \times 11^{17} \text { C. G. S. E. S. } \\
& N_{\mathrm{e}}=3 \times 10^{15} \quad p_{\mathrm{c}}=1 \\
& 11-N_{\mathrm{e}} F_{\mathrm{c}}=0,23 \times 10^{18} \text { C. G. S. E.S. } \\
& N_{\mathrm{e}}=3 \times 10^{15} \quad p_{\mathrm{c}}=3 \\
& 14-N_{\mathrm{c}} F_{\mathrm{c}}=0,25 \times 10^{18} \text { C. G. S. E. S. } \\
& N_{\mathrm{e}}=4 \times 10^{15} \quad p_{\mathrm{c}}=2 \text {. }
\end{aligned}
$$

[Comparison of $\mathrm{H}_{\alpha}$ and $\mathrm{H}_{\beta}$ for various values of the product $N_{\mathrm{e}} F_{\mathrm{c}}$

Curves $6 N_{\mathrm{e}} F_{\mathrm{c}}=0.79 \times 10^{17}$ C. G. S. E. S.

$N_{\mathrm{e}}=2 \times 10^{15} \mathrm{~cm}^{-3} \quad p_{\mathrm{c}}=2$

$9 N_{\mathrm{e}} F_{\mathrm{c}}=0.79 \times 10^{17}$ C. G.S. E. S

$N_{\mathrm{e}}=3 \times 10^{15} \mathrm{~cm}^{-3} \quad p_{\mathrm{c}}=1$

$11 N_{\mathrm{e}} F_{\mathrm{c}}=0.23 \times 10^{18}$ C. G. S. E. S.

$N_{\mathrm{e}}=3 \times 10^{15} \mathrm{~cm}^{-3} \quad p_{\mathrm{c}}=3$

$14 N_{\mathrm{e}} F_{\mathrm{c}}=0.25 \times 10^{18}$ C. G. S. E. S

$\left.N_{\mathrm{e}}=4 \times 10^{15} \mathrm{~cm}^{-3} \quad p_{\mathrm{c}}=3.\right]$

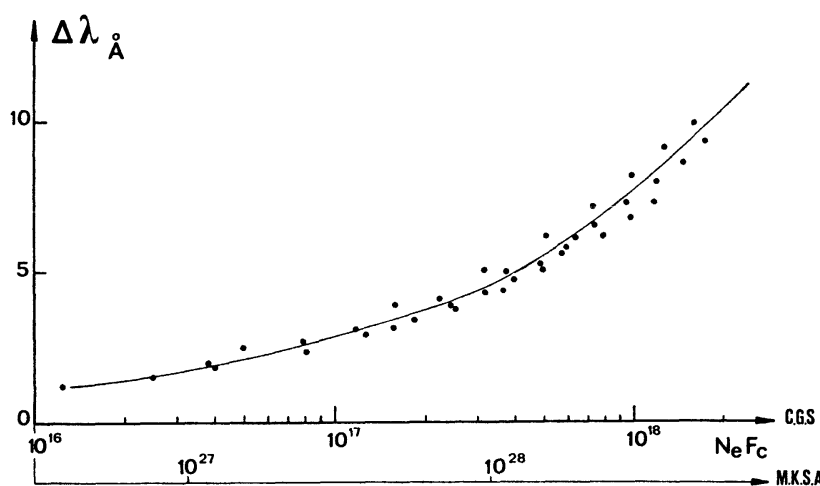

FIG. 6. - Demi-élargissement à moitié du maximum pour $\mathrm{H}_{\beta}$ en fonction du produit $N_{\mathrm{e}} F_{\mathrm{c}}$ et $1 \leqslant p_{\mathrm{c}} \leqslant 4$.

[Half-max-intensity half-width for $\mathrm{H}_{\beta}$ as a function of the product $N_{\mathrm{e}} F_{\mathrm{c}}$ and with $1 \leqslant p_{\mathrm{c}} \leqslant 4$.]

La théorie utilisée suppose que le champ auquel est soumis l'atome émetteur n'est pas trop élevé. Ceci donne la limite supérieure de validité de la théorie (voir Fig. 7).

Une discussion détaillée du domaine de validité de la théorie est donnée dans [1]. En particulier la théorie ne peut s'appliquer sur la partie lointaine des ailes. En pratique nous n'avons pas développé les calculs audelà de $\Delta \omega>3(\Delta \omega)_{\text {demi-largeur }}$, domaine dans lequel la contribution du champ macroscopique n'est nulle part négligeable dès que $p_{\mathrm{c}}>1$. 


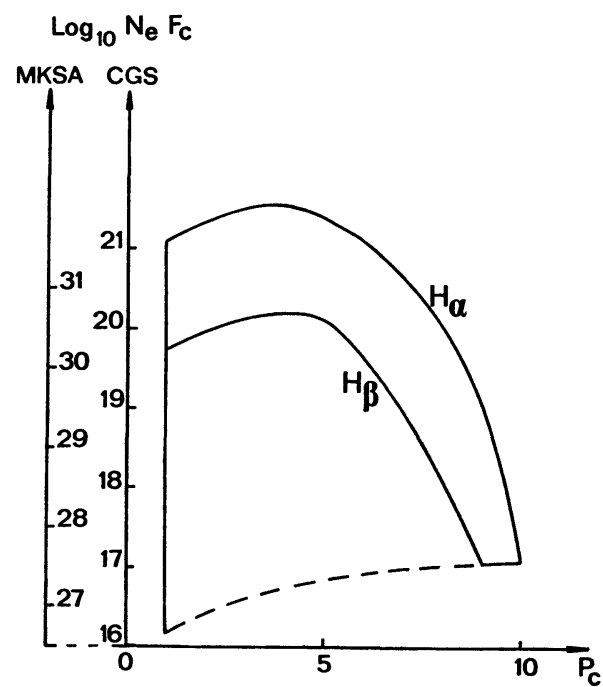

Fig. 7. - Diagramme de validité de la théorie en coordonnée $j_{\mathrm{e}}$, $p_{\mathrm{c}}$ avec $T_{\mathrm{e}}=10000 \mathrm{~K}$. Le trait pointillé correspond à $N_{\mathrm{e}}=10^{15} \mathrm{~cm}^{-3}$.

[Validity of theory in a $\left(j_{\mathrm{e}}, p_{\mathrm{c}}\right)$ diagram with $T_{\mathrm{e}}=10000 \mathrm{~K}$ dotted line corresponds to $N_{\mathrm{e}}=1015 \mathrm{~cm}^{-3}$.]

4. Application à la décharge couronne. - Les résultats précédents ont été appliqués à l'étude de la décharge entre une pointe portée à une tension positive et un plan (décharge couronne). Nous avons utilisé de l'oxygène en $y$ ajoutant des traces d'hydrogène. Le régime de décharge est un régime dans lequel les mécanismes de croissance du courant conduisant à un filament d'arc apparaissent. On trouvera une description plus détaillée des conditions expérimentales dans [7].

La comparaison des élargissements obtenus avec les résultats théoriques précédents a permis, compte tenu des élargissements instrumental et Doppler, de déterminer l'évolution de $N_{\mathrm{e}} F_{\mathrm{c}}$ en fonction du temps.

Ayant une valeur de la mobilité électronique $\mu_{\mathrm{e}}$ on obtient la densité de courant $j_{\mathrm{e}}$ en fonction du temps (voir Fig. 8).

Ajoutons que d'autres considérations en (particulier une méthode de simulation [8]) permettent d'évaluer $F_{\mathrm{c}}$ donc $N_{\mathrm{e}}$ et $p_{\mathrm{c}}$. On vérifie donc que $p_{\mathrm{c}}$ se trouve bien dans le domaine de validité de la théorie.

Connaissant le courant $I$, on peut à partir de $j$ obte-

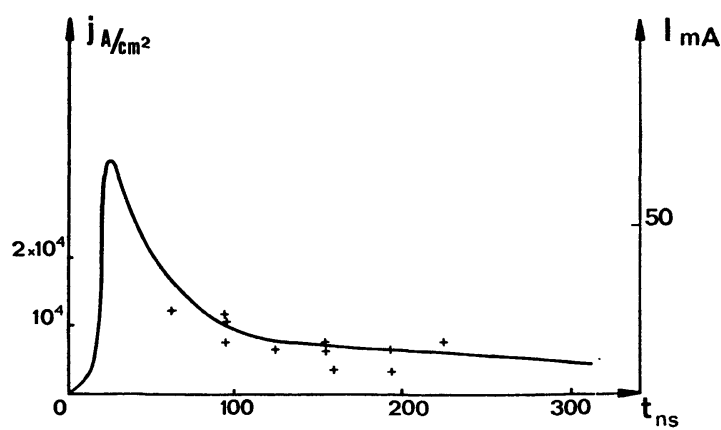

Fig. 8. - Densité de courant $(+)$ en fonction du temps et courant en fonction du temps pour un essai dans l'oxygène (avec traces d'hydrogène) à une pression de 270 torr.

[Experimental current density $(+)$ and current as functions of time in oxygen (hydrogen traces added) at a pressure of 270 torr.]

nir la valeur de surface de la section droite de la décharge. Ce paramètre difficile à mesurer, étant donné sa petitesse, a une grande importance pour déterminer les mécanismes de transition vers l'arc. En effet il conditionne les vitesses d'échange entre le milieu ionisé et l'extérieur. Dans le cas présenté ici, on trouve une valeur moyenne de $S=2,5 \times 10^{-10} \mathrm{~m}^{2}$ soit un rayon de l'ordre de $9 \mu \mathrm{m}$. Cette valeur est caractéristique ; elle dépend peu du mode de répartition radiale des charges. En effet, le rayon tiré à l'aide d'un profil théorique qui tient compte d'une répartition radiale gaussienne des charges a une valeur similaire.

5. Conclusion. - Dans cet article nous avons exposé une conséquence pratique intéressante de l'analyse Stark obtenue en présence d'un champ macroscopique $F_{\mathrm{c}}$. En effet, bien que l'élargissement dépend principalement de deux paramètres différents $N_{\mathrm{e}}$ et $F_{\mathrm{c}}$, nous avons montré que l'élargissement détermine sensiblement le produit $N_{\mathrm{e}} F_{\mathrm{c}}$, c'est-à-dire, la densité de courant connaissant la mobilité.

Cette analyse permet donc d'appliquer une méthode nouvelle de diagnostic aux milieux ionisés produits par les décharges prédisruptives en ajoutant des traces d'hydrogène.

Une telle méthode pourrait s'avérer utile chaque fois où l'on cherche à préciser des taux de production (chimie des plasmas), car ceux-ci sont liés à la densité de courant.

\section{Bibliographie}

[1] Bastien, F., Marode, E., J. Quant. Spectros. Radia. Transfer. 70 (1977) 4, 453.

[2] Griem, H. R., Kolb, A. C., Shen, K. Y., Phys. Rev. 116 (1959) 1, 4; (N. R. L. report 5455).

[3] N'Guyen Hoe, Drawing, H. W., Herman, L. (Rapport C. E. A. R 3057 (1966); J. Quant. Spectros. Radia. Transfer. 7 (1967) 429.

[4] Hooper, C. F. jr, Phys. Rev. 165 (1968) 215 ; Phys. Rev. 169 (1968) 193.
[5] Jensen, V. O., Proceedings of 7th Intern. Conf., on Phenomena in Ionized Gases (Beograd) 1966, Vol. 2, 553.

[6] Deutsch, C., Bekefi, G., Phys. Rev. 14 (1976) 854.

[7] Bastien, F., Fertil, B., Marode, E., J. Phys. D 9 (1976) L 155 .

[8] Marode, E., J. Appl. Phys. 46 (1975) 2005 ; J. Appl. Phys. 46 (1975) 2016. 